\title{
Convex side muscular activity and progression of scoliotic curve
}

\author{
Gundars Rusovs*, A Vetra \\ From 7th International Conference on Conservative Management of Spinal Deformities \\ Montreal, Canada. 20-22 May 2010
}

\section{Introduction}

Muscular asymmetry is considered as important etiological factor of idiopathic scoliosis. The present study analyses pathomechanisms leading to progression of scoliotic deformation as the result of asymmetric paraspinal muscular activity irrespective of the primary trigger.

\section{Material and methods}

Review of articles published in Medline, Science Citation Index and other sources on topic scoliosis and paraspinal muscular activity whose content provided data on the biomechanical approach associated with formation of spinal curvatures.

\section{Results}

Increased paraspinal muscular activity at convex side of scoliotic curve is well known and documented finding. Main directions of research are related to structural changes in muscles and regulation of muscular contractility. In most cases the myopathic changes are considered as the consequence of the postural deformity. Very little information is available on the mechanisms by which such an imbalance could take place.

\section{Discussion}

One of the main tasks of paraspinal muscles is stabilization of upright spine. Everyday activities as walking and running are tending to accelerate forward the head, arms and thorax (HAT) around thoracolumbar junction at sagital plane. The stability of thoracic spine is achieved by first class lever where frontal part of vertebra is loaded by weight of HAT, intervertebral disc is fulcrum and paraspinal muscles play the role of effort. In scoliotic spine position of load on vertebra is shifted to concave side, so unilateral activation of convex side muscles is biomechanical consequence. Unilateral activity of paraspinal muscles creates rotational moment between vertebras. During dynamic loading, upper vertebra becomes fixed point. Rotating moment around it is created tending to rotate vertebra below to convexity. The mobilization of ribs as lever arm is only available method to prevent rotation, since unilateral paraspinal activity has no muscular antagonists. System is stabile till strength of ribs yields to banding pressure and vertebral rotation becomes possible. Buckling of ribs under cumulative banding forces is related to the period of rapid growth in accordance with the Hueter-Volkmann law.

\section{Conclusion}

Progression of scoliosis can be considered as side effect from dynamic stabilization of scoliotic thoracic spine by paraspinal muscles because of rotating moment created by unilateral muscular activity.

The speed of progression depends on the strength of ribs.

Published: 10 September 2010

doi:10.1186/1748-7161-5-S1-O23

Cite this article as: Rusovs and Vetra: Convex side muscular activity and

progression of scoliotic curve. Scoliosis 2010 5(Suppl 1):O23.

Riga Stradins University, Riga, Latvia

Full list of author information is available at the end of the article 